Military Technical College

Kobry El-Kobbah,

Cairo, Egypt.

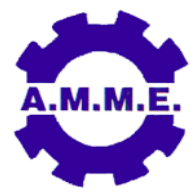

$16^{\text {th }}$ International Conference on Applied Mechanics and Mechanical Engineering.

\title{
HOT-PRESSED ELECTROSPUN MWCNTS/CARBON NANO FIBRIL COMPOSITES: POTENTIAL APPLICATIONS FOR BREAKING PADS AND JOURNAL BEARING
}

\author{
A. A. Ali ${ }^{1}$ A. R. Eldesouky ${ }^{2}$ and S. H. Zoalfakar $^{3}$
}

\begin{abstract}
Most of the mechanical and tribological properties of electrospun fiber fabrics are of paramount importance to their utility as components in a large number of applications. Post-spin heat treatment assisted with pressure was used to modify the properties of the electrospun fabrics and to activate the high surface energy. HRTEM showed a formation of graphitic structure with $9.5 \mathrm{~nm}$ crystallite size which calculated from Raman breathing modes. Hot-pressed MWCNTs/Carbon fabrics with $130 \mathrm{~nm} \pm$ $32 \mathrm{~nm}$ fibril diameter showed an outstanding flexibility and strength. Also, adding MWCNTs improved tensile strength (from 40 to 60MPa), coefficient of friction (from 0.5 to 0.15 ) and abrasive mass loss (as low as $0.2 \mathrm{mg}$ has been achieved). The hotpressed electrospun MWCNTs/Carbon nanofibril composite fabrics can be used, by controlling MWCNTs wt\%, as a good candidate for both journal bearings (C.O.F. below 0.4 is required) and braking pads (C.O.F. from 0.4 to 0.7 are required).
\end{abstract}

\section{KEY WORDS}

Electrospinning, SEM, HRTEM, Heat treatment, Mechanical properties, Tribological

Assistant professor, Dept. of Design Engineering, Zagazig University, Egypt.

Professor, Dept. of Prod. Engineering, Menoufiya University, Egypt.

Ph.D. Student, Dept. of Mech. Engineering, the Higher Tech. Institute (HTI) Egypt. 


\section{INTRODUCTION}

Electrostatic fiber formation, or electrospinning, offers a particularly simple and robust method to create polymeric nanofibers of various morphologies and sizes, inexpensively and in large quantities. In electrospinning, a viscoelastic fluid is charged so that a liquid jet is ejected from the surface of the fluid (typically supplied by a needle or spinneret) and accelerated by an electric field towards a collector, typically a grounded plate, thus creating a nonwoven fiber mat or membrane [1]. Ali AA et al. [2] activated the high surface energy of the electrospun PAN nano fibers as a cohesive bond to build a firm strong precursor carbon nano fibers fabric. Also, it uses the high degree of entanglement inside the nano fibrous structure to gain a higher degree of flexibility in the produced fabric. Optimum electrospinning conditions by using response surface methodology (RSM) have been recalled from previous work to produce MWCNTs/PAN nanofibril composites.

The as electrospun fabrics have been heat treated through two steps; stabilization at $220^{\circ} \mathrm{C}$ and, carbonization at $380^{\circ} \mathrm{C}$ [3]. Polyacrylonitrile (PAN) and polyvinylpyrrolidone (PVP) solutions incorporated with multi wall carbon nanotubes (MWCNTs) and NiZn ferrite (Ni0.6Zn0.4Fe2O4) nanoparticles were electrospun at various weight percentages, and then thermal conductivity values of these nanocomposite fibers were determined using a comparative method. During the electrospinning process, system and process parameters, such as concentrations, applied voltage, tip-to-collector distance, and pump speeds were optimized to receive the consistent nanocomposite fibers [4]. Mesopore-enriched activated carbon nanofiber (ACNF) mats are produced by incorporating vanadium (V) oxide (V2O5) into polyacrylonitrile (PAN) via electrospinning, and their electrochemical properties areinvestigated as an electrode in supercapacitors [5].

Yeon-Joo Kim et al. [6] indicated that the non-woven PAN separator has many advantages over the PE separator, which, in turn, contribute to superior cell performances. Therefore, the PAN nonwoven membrane can be the most promising candidate for separators of the next generation of lithium-ion battery if the problems to be stated will be solved. Zhang et al. [7] found that the sliding velocity plays significant roles on the tribological characteristics by influencing the interface temperature and strain rate of the polyether-ether-ketone surface layer involved in the friction process, and the applied load influences the tribological performance by varying the strain range in the surface layer. Electrospun nanofibers have been reported to exhibit some remarkable increases in elastic stiffness and yield stress for fibers below a critical diameter, whose value varies from material to material [8]. The origin of these increases in fiber mechanical properties remains a topic of some debate. Regardless of the diameter-dependent changes in fiber properties, the asspun mats tend to exhibit consistently low yield stresses (typically 0.5-3 MPa), Young's moduli (typically 20-60 MPa) and toughnesses (typically 0.5-2 MJ/m3) [9]; as-spun untreated nanofiber mats also exhibit low mechanical resilience $(<50 \%)$ even at small strains of less than $0.02 \mathrm{~mm} / \mathrm{mm}$ [10].

Recently, several research groups have demonstrated significant improvements to the Young's modulus and yield stress of electrospun polymeric fiber mats by various forms of post-spinning techniques such as thermal annealing [11], mechanical drawing [12], hot pressing [13], and solvent vapor treatment [14]. Subjecting a semi- 
crystalline polymer fiber to heat treatment at a temperature above the crystallization temperature (Tc) of that fiber, but below the equilibrium melting temperature (Tm) of the polymer, can cause the melting of small, imperfect crystals, and the formation of larger, more perfect crystals within the fibers, thus creating a stiffer and tougher matrix [15]. With some amorphous polymer nonwovens, annealing has been shown to allow air or gas pockets within the fibers to diffuse out, creating stronger more uniform fibers [16].

Investigation of the tribological properties of textiles and fabrics is not unprecedented; Derler et al. measured the friction coefficient and hardness of conventional textiles in contact with human skin equivalents [17], and Gerhardt et al. measured the frictional properties and contact pressure of skine fabric interactions, for example [18]. Guo-ming Lin et al. [19] indicated that the wear rates of the hybrid composites decreased with the increasing applied load and sliding distance under water lubrication, low friction coefficient and low wear rate could be achieved at high sliding velocity. Matthew M. Mannarino [20] studied The mechanical and tribological properties of electrospun PA 6(3)T fiber mats and concluded that the average fiber diameter of $463 \pm 64 \mathrm{~nm}$. Post-spin thermal annealing was used to modify the properties of the fiber mats. Morphological changes, in-plane tensile response, friction coefficient and wear rate were characterized as functions of the annealing temperature. The Young's moduli, yield stresses and toughnesses of the nonwoven mats improved by two- to ten-fold when annealed slightly above the glass transition temperature, but at the expense of mat porosity. The coefficient of friction and the wear rate decrease by factors of two and ten, respectively, under the same conditions. T. Policandriotes [21] concluded that the goal in aircraft brake friction materials is to maintain a high and stable friction level and reduce oxidative wear. $A$ high stable friction level in the range of $0.4-0.7$ is desired in all brake conditions. Wear in $\mathrm{C} / \mathrm{C}$ is due mostly to oxidation of the carbon fiber and carbon matrix. This oxidation increases abrasive wear and reduces friction. A selection of nanoadditives is therefore impregnated into the open porosity of commercial $\mathrm{C} / \mathrm{C}$ to reduce oxidation and increase friction.

In this paper, MWCNTs has been used to improve the mechanical and tribological properties of as electro-spun PAN nano fabrics. Also, hot-pressing technique has been introduced to enchance prosity and to reduce fiber diameter as well as to activate surface energy a compared with nanofibers.

\section{EXPERIMENTAL WORK}

\section{Materials}

Polyacrylonitrile (PAN) of $150000 \mathrm{~g} / \mathrm{mol}$ molecular weight from Aldrich catalog no. (181315) was used with10\% weight concentration in dimethylformamide (DMF) to form a polymer solution after hot stirring for $3 \mathrm{hrs}$ at $60 \mathrm{oC}$ to ensure a complete solubility. MWCNTs size from Aldrich (MWCNT: O.D. $=40-60 \mathrm{~nm}$, I.D. $=5-10 \mathrm{~nm}$, $\mathrm{L}=0.5-500 \mu \mathrm{m}$ ) has been well dispersed (after 24 sonication hours) inside the PAN/DMF polymer solution within five different concentrations from $1 \%$ to $5 \%$ by weight. 


\section{Electrospinning}

The electrospinning setup has been assembled in our lab. The PAN/DMF-CNT polymer solution was poured to fill a clean syringe of $10 \mathrm{ml}$ volume. The syringe was connected to a metal tube of orifice inner diameter $0.9 \mathrm{~mm}$. The metal tube was connected to the power supply at $25 \mathrm{kV}$ of positive potential. A metal screen collector of $15 \times 15 \mathrm{~cm}$ dimensions was centered horizontal at a $20 \mathrm{~cm}$ distance away from the orifice of the metal tube and covered with aluminum foil. The electrospun fibers were collected for about 8 hours.

\section{Heat Treatment}

\section{Stabilization}

About $2 \mathrm{~cm}$ from each side of the collected fiber mat was trimmed then the fabric was placed in between two aluminum plates of $110 \times 105 \mathrm{~mm}$ dimensions and $10 \mathrm{~mm}$ thickness after being covered with aluminum foil. The mold with the fabric in between was placed in a hot press set to reach $220 \mathrm{oC}$ with no applying pressure for 1 hour until the plate's temperature reached the maximum set temperature. Then 1 metric ton was applied for another one hour. The hot press was then allowed to cool down for another 1 hour while keeping the pressure on until it reaches $100 \mathrm{oC}$ then the pressure was released completely from the fabric except the weight of the upper aluminum plate until it cooled down to room temperature. Also, the morphological behavior of the nano fibers before and after hot pressing has been investigated using SEM.

\section{Carbonization}

Hot pressing in isolated medium from environmental air for 3 hour in between single plate and double aluminum foils with $5000 \mathrm{Kg}$ at $380^{\circ} \mathrm{C}$ pressure released after cool down to $100{ }^{\circ} \mathrm{C}$.

\section{Characterization}

\section{Electron microscopy}

Scanning electron microscopy (SEM, JEOL JSM-5600LV) in the Central Laboratory for Elemental and Isotopic Analysis (Atomic Energy Authority Nuclear Research Center, Egypt) was used to characterize the fiber morphology, average diameter and its distribution of the resultant PAN/MWCNTs nanofibers. Transmission electron microscopy (HTEM) (JEOL model JEM 2100) in Petroleum research Institute, Egypt) was used to investigate the morphologies of the pristine and the MWNTs as well as the alignment of the MWNTs in the resulting PAN/MWNTs composite nanofibers.

\section{Raman spectroscopy}

The Raman spectra were recorded with a Raman spectrometer (FT/IR-6300typeA), and the spectral resolution was $16 \mathrm{~cm}^{-1}$. Central Metallurgical Research and Development Institute (CMRDI), Egypt.

\section{Tensile test}

Five tensile samples of approximately $60 \mathrm{~mm}$ length and $5 \mathrm{~mm}$ width have been cut from the hot-pressed nano fibroses fabric. Each sample has been weighted by 3 
digits balance. Zwick UTM machine with $500 \mathrm{~N}$ maximum load cell and $1 \mu \mathrm{N}$ accuracy has been used.

\section{Tribology characterizations}

Friction force measurements were taken at a series of normal forces ranging from 0.25 to $3.5 \mathrm{~N}$, at a testing speed of $0.14 \mathrm{~m} / \mathrm{s}$. (GUNT HAMBURG TM 260.03 MODULE PIN ON DISC) used in the abrasive wear testing experiments. The effective radius of the disk was $40 \mathrm{~mm}$ and testing was performed at 60rpm. Test samples were prepared by carefully cutting out $10 \mathrm{~mm}$ diameter circles from an electrospun fabric, stabilized and carbonized and attaching them to the adhesive side of a $10 \mathrm{~mm}$ diameter Polyken ${ }^{\circledR} 339$ duct closure foil. This prevents the fabrics from bunching up or shifting during testing. Number of cycles has been used 60, $120,300,480,720,1020$ and 1200 with all 18 samples.

\section{RESULTS AND DISCUSSION}

\section{Morphology Characterizations}

Figure 1 shows SEM pictures of the fiber diameters and its patterns for the as electrospun PAN, stabilized and hot-pressed one. It has been found that; the fiber kept its morphological behavior after both stabilized and hot pressing. Also, the measured average fiber diameter shown in Fig.1a found to be $194 \mathrm{~nm} \pm 34 \mathrm{~nm}$ for the as-electrospun, $145 \pm 38 \mathrm{~nm}$ for stabilized as shown in Fig. $1 \mathrm{~b}$ and $140 \mathrm{~nm} \pm 50 \mathrm{~nm}$ for the hot pressed as shown in Fig.1c. Also, as shown in Fig. 1d MWCNTs/PAN average diameter of nano fibril composite found to be $183 \mathrm{~nm} \pm 32 \mathrm{~nm}$ for aselectrospun, 151 $\pm 32 \mathrm{~nm}$ for stabilized as shown in Fig.1e and $130 \mathrm{~nm} \pm 32 \mathrm{~nm}$ for the hot pressed as shown in Fig.1f. The electrospun MWCNTs/PAN fibril composite fabric was observed by high resolution transmission electron microscopy (HRTEM). HRTEM images show MWCNTs bundle with diameter of $14 \mathrm{~nm}$ wrapped by PAN wass aligned along the fiber axis (Fig.2a \&b). Fig.2c proved a formation of 2-D graphitic structures of $0.333 \mathrm{~nm}$ lamella thickness. Also Fig.2d diffraction pattern of the sample shows a high level of crystallinity for the hot-pressed nanofibril composite fabrics.

\section{Raman Spectra of Electrospun MWCNTs/PAN Nanofibril Composites}

Raman spectroscopy was conducted on electrospun MWCNTs/PAN nano fibril composite fabrics to study the interaction between MWCNTs and PAN during hot pressing. In Fig.3a the spectra of the electrospun PAN nano fiber diameters without any MWCNTs reinforcements before and after heat treatments showed the increase in the carbon contents within the sample as the heat treatments took place. For the stabilized and carbonized steps a two peaks at 1360 and $1580 \mathrm{~cm}^{-1}$ approximately show $D$ and $G$ breathing modes respectively from which crystallite size can be calculated. An increase in the $\mathrm{G}$ band intensity was detected in the carbonized step, which indicates more ordered structure than the stabilized one. From Fig.3b shows the evident of the presence of MWCNTs in the electrospun PAN nanofibers by detecting a larger peak as MWCNTs wt\% increases compared with as electrospun pure PAN shown in Fig.3a. Also, Fig.3b for stabilized fabrics showed a peak started 
to appear as more heat treatment took place which indicates a higher order structure more than as electrospun one.

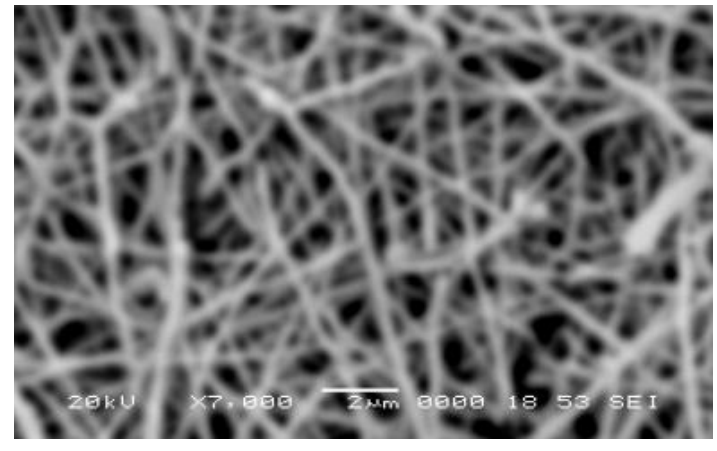

(a)

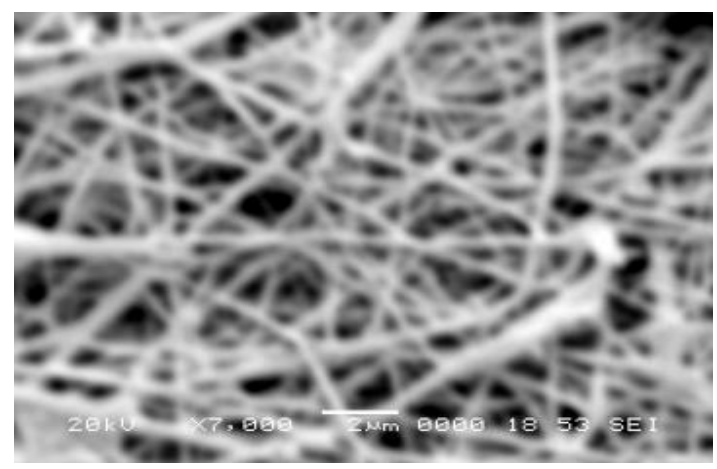

(c)

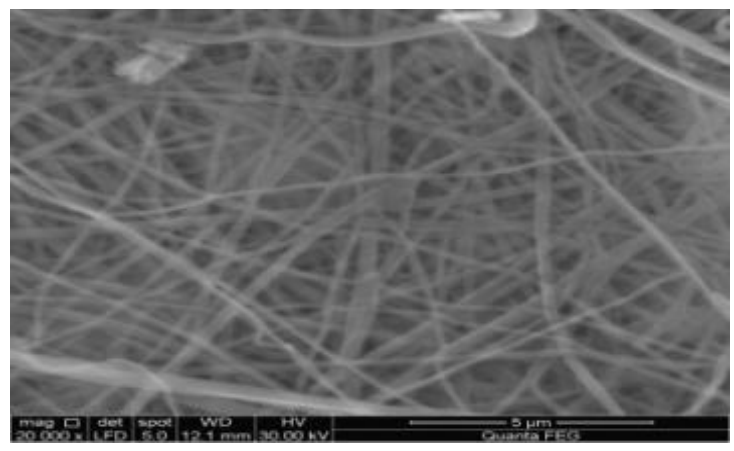

(e)

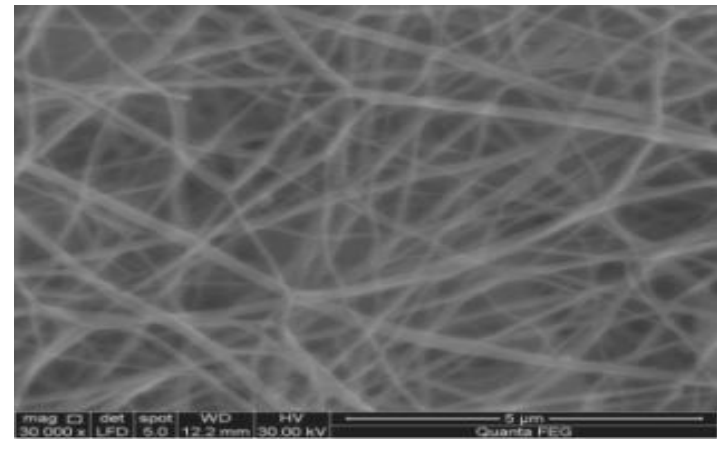

(b)

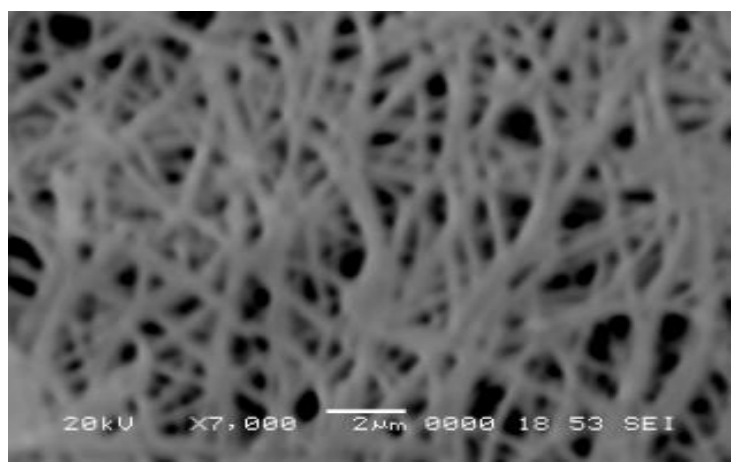

(d)

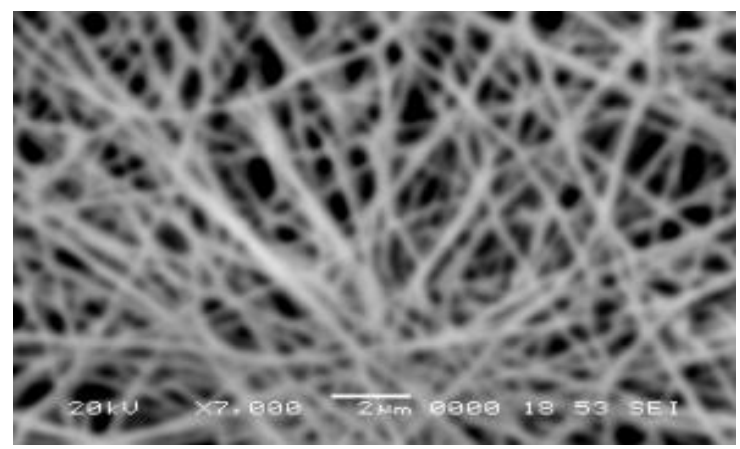

(f)

Fig. 1. SEM micrographs of composites CNF: (a) Pure PAN As electrospun, (b) Pure PAN stabilized, (c) Pure PAN hot pressed, (d) $5 \mathrm{wt} \%$ MWCNT in PAN As electrospun, (e) 5 wt\% MWCNTs in PAN stabilized ,(f) 5 wt\% MWCNTs in PAN hot pressed. 


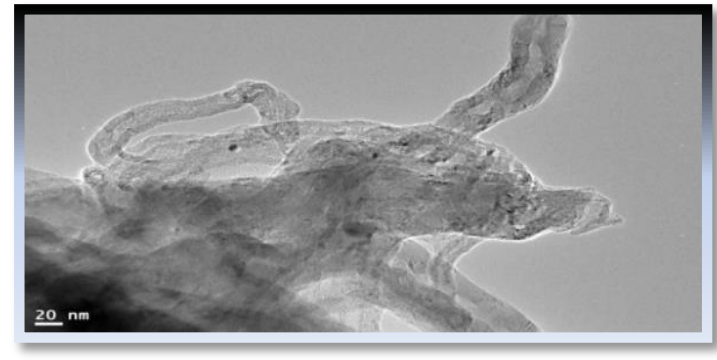

(a)

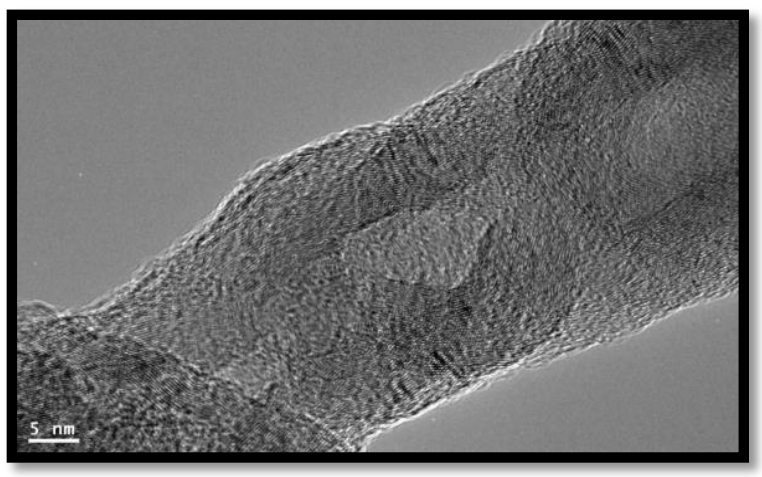

(c)

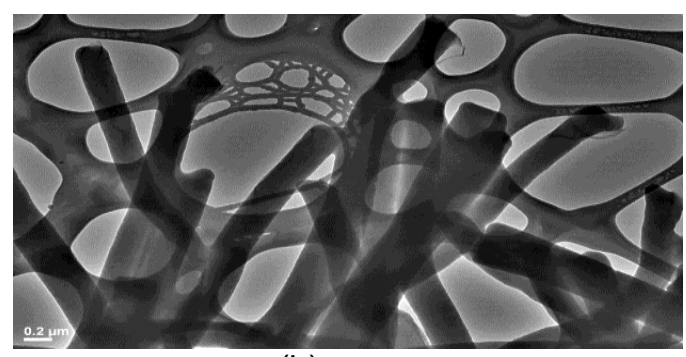

(b)

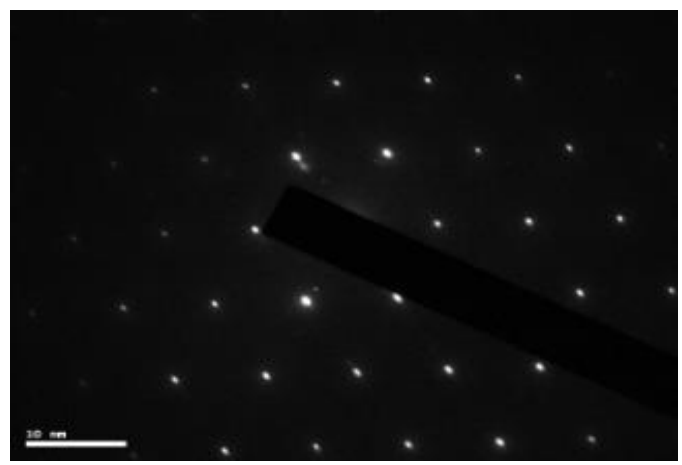

(d)

Fig. 2. Transmission electron micrograph of (a \&b) electrospun PAN/MWCNTs Nanofiber, (c) Formation of $0.333 \mathrm{~nm}$ graphite lamells for hot pressed PAN/MWCNTs samples and (d) MWCNTs diffraction pattern.

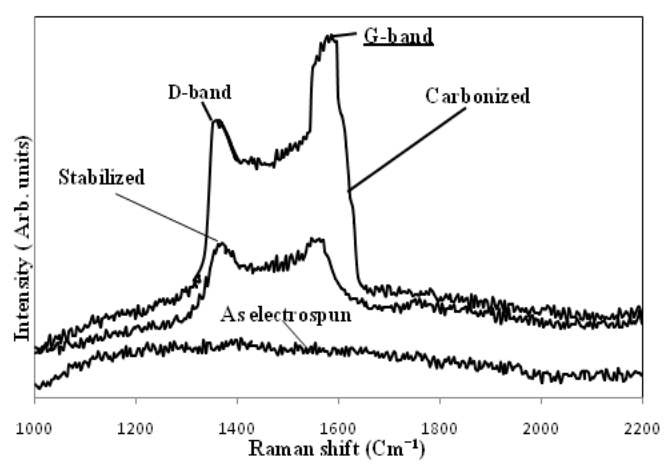

(a)

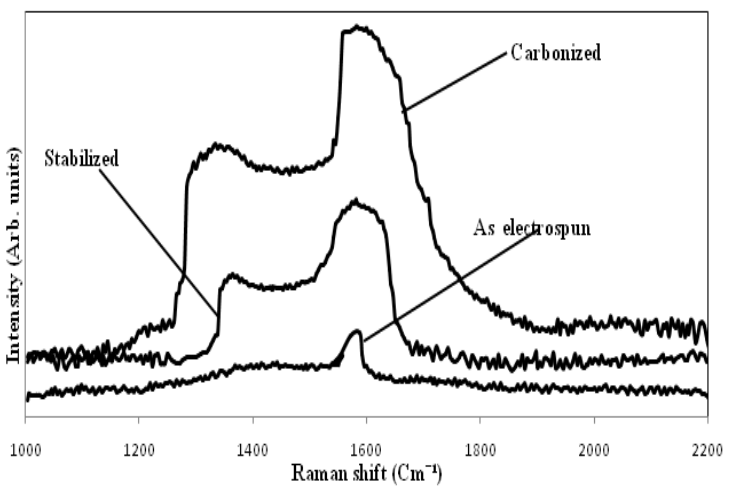

(b)

Fig. 3. Raman spectra of (a) Raman Results for the Electro-Spun PAN Nano Fibers Before and After Heat Treatments and (b) PAN/MWCNTs nanofiber. 


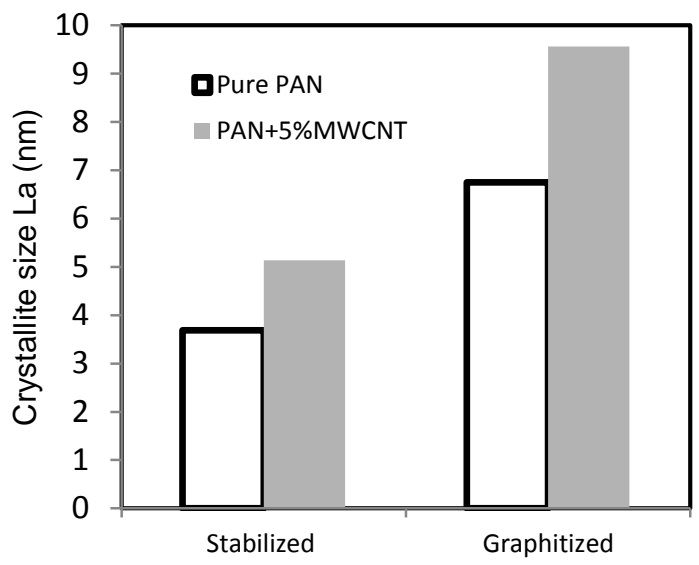

Fig. 4. Crystallite size for the as electrospun, carbonized and graphitized Electrospun.

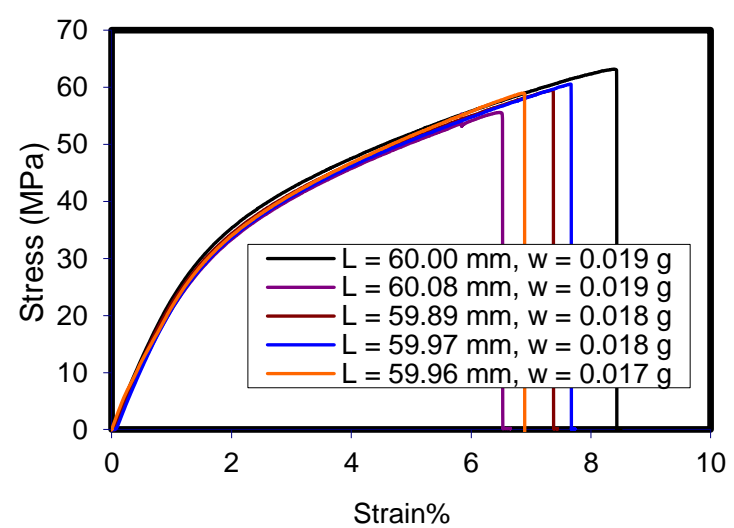

(a)

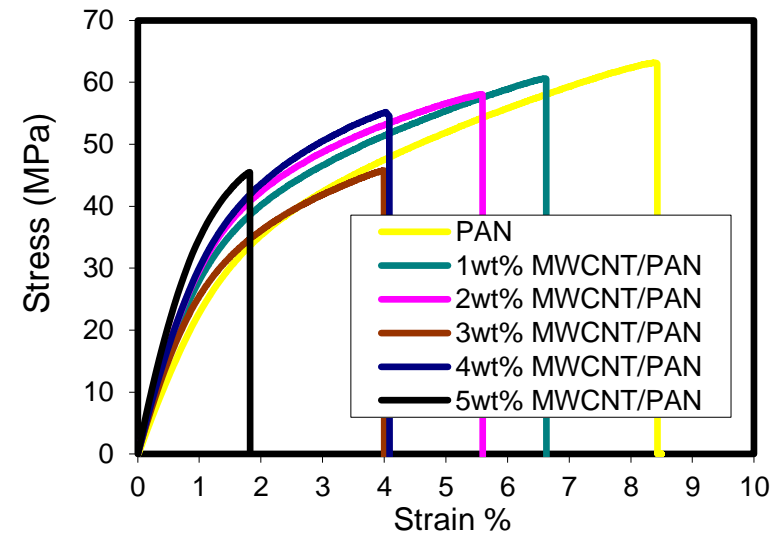

(b)

Fig. 5. Stress-strain curve for (a). hot pressed electro-spun PAN nano fiber fabric, (b).Hot pressed electrospun MWCNTs/PAN nano fibril composites.

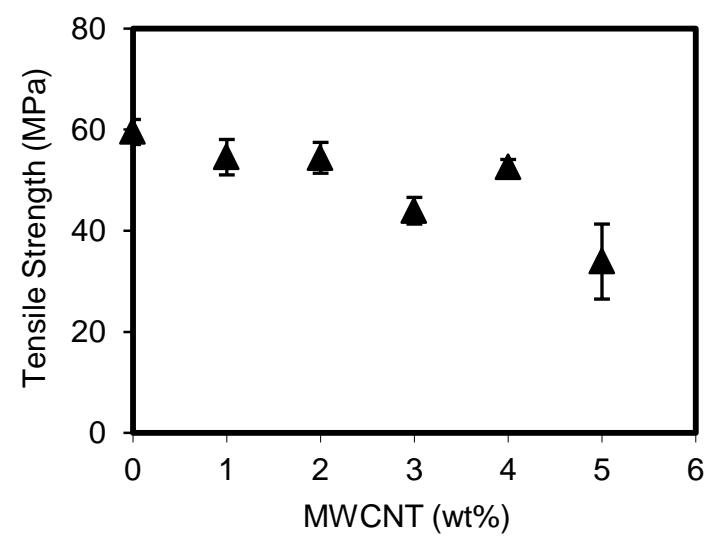

Fig. 6. Tensile strength of hot-pressed MWCNTs/PAN nano fibril composite fabrics. 


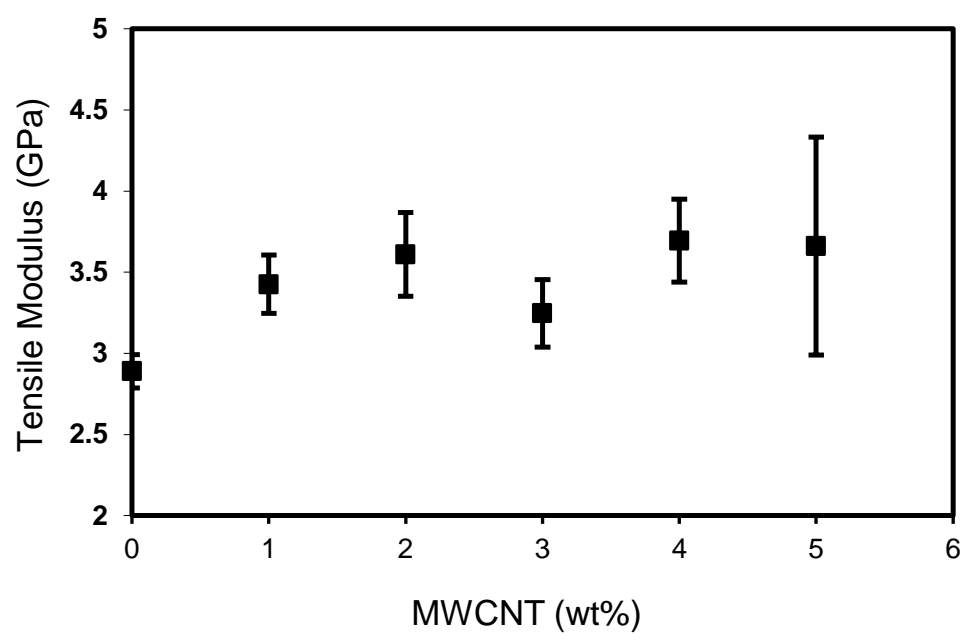

Fig. 7. Tensile modulus of hot-pressed MWCNTs/PAN nano fibril composite fabrics.

The crystallite size was also calculated by using the empirical formula, $\mathrm{La}=\mathrm{C}$ $(\lambda)^{*}(\mathrm{Ig} / \mathrm{ld})$, where $\mathrm{La}$ is the crystallite domain's size and $\mathrm{C}$ is a function of the laser wavelength $(C(\lambda)=4.4 \mathrm{~nm}), \mathrm{I}_{D}$ and $I_{G}$ are the $D$ and $G$ peak intensities [22]. The results are listed in (Table 1) as observed in Fig.(4), the crystalline domain's size for pure PAN increased from 3.69 to 6.75 at stabilization and carbonization. Also, the crystalline domain's size for CNT/PAN composite fiber increased from 6.14 to 9.56 $\mathrm{nm}$.

Table 1: Crystallite size for the as electrospun, carbonized and graphitized Electrospun PAN and PAN/MWCNTs nano composite fibrils.

\begin{tabular}{|l|c|c|}
\hline \multirow{2}{*}{ Composition } & \multicolumn{2}{|c|}{ La $(\mathrm{nm})$} \\
\cline { 2 - 3 } & stabilized & Carbonized \\
\hline Pure PAN & 3.97 & 5.74 \\
\hline PAN+5wt\% MWCNT & 5.14 & 9.56 \\
\hline
\end{tabular}

\section{Mechanical Properties Results}

The stress-strain behavior of five samples has been tested and shown in (Fig. 5a), the elastic-plastic behavior of the samples was similar to the behavior of many other materials such as metals and polymers and was not similar to the behavior of commercial in-plane random fiber fabrics that affected by the orientation of their fibers in the applied force direction. The measured maximum and minimum tensile strength for the hot pressed samples found to be $63.2 \mathrm{MPa}$ and $55.6 \mathrm{MPa}$ simultaneously while the measured average tensile strength for the as electrospun fabric samples found to be equal $8.7 \mathrm{MPa}$ with $3 \mathrm{MPa}$ standard deviation. Also, (Fig. 5b) hot-pressed electrospun MWCNTs/PAN nanofibril composite fabrics showed an elastic characteristic with firm and strong behavior of $60 \mathrm{MPa}$ fabric strength. The increase in MWCNTs decreases tensile strength due to more delamination and brittle fractures that has been detected as shown in Fig.6. Fig.7 shows that as the MWCNTs\% by weight increases the modulus of elasticity value increases. Also show that the 
measured modulus values fond to be in the range between 3-4 GPa for the hot pressed nano fibril composite fabrics is the measure of the physical bond between nano fibers and the fiber characteristics itself.

\section{Tribology of Electrospun Fabrics}

Electrospun nano fiber and nanofibril composite fabrics are typically fragile and susceptible to wear and delamination even under conditions of gentle handling. This is a concern for the post-spin processing as well as the end-use of such mats and could be a critical limitation to their service lifetime. To quantify this behavior, the coefficient of friction was measured using a standardized testing rig (GUNT HAMBURG TM 260.03 MODULE PIN ON DISC) for each of nanofiber fabrics, aselectrospun, stabilized and hot-pressed. A plot of the coefficient of friction vs. the normal force is shown in Fig. (8a-8c); note that there is a modest decrease in the coefficient of friction with increasing normal force at low loads, but that above $2 \mathrm{~N}$, the coefficient of friction is approximately constant, especially for the samples at stabilized or/and hot-pressed. Also the coefficient of friction decrease with increasing addition of MWCNTs. Mean friction coefficients at $1 \mathrm{~N}$ load decreases from 1.6 for as electrospun PAN nanofiber Fig $8 \mathrm{a}$ to 1 for stabilized one Fig. $8 \mathrm{~b}$ then to 0.7 for hotpressed fabric Fig.8c. A plot of the abrasive mass loss vs. the number of cycles for $200 \mathrm{~g}$ applied load is shown in Fig. 9 for 60, 120, 300, 480, 720, 1020, and 1200 cycles. Note that; there is a modest increase in the abrasive mass loss with increasing number of cycles, but the abrasive decrease with additive MWCNTs wt\% increase for example; abrasive mass loss are 2.7 and $1.5 \mathrm{mg}$ respectively for Pure PAN nano fiber and 5wt\% MWCNTs/PAN nano fibril fabric, Also, comparison between Fig.9a \& Fig.9b showed decrease in the abrasive mass loss for as electrospun to hot-pressed from $1.5 \mathrm{mg}$ to $0.65 \mathrm{mg}$ at $5 \mathrm{wt} \%$ MWCNTs/PAN nano fibril composite fabrics. The hot-pressed electrospun MWCNTs/Carbon nanofibril composite fabrics showed an outstanding behavior that can be used, by controlling MWCNTs wt\%, as a good candidate for both journal bearings (C.O.F. below 0.4 is required) and braking pads (C.O.F. from 0.4 to 0.7 are required).

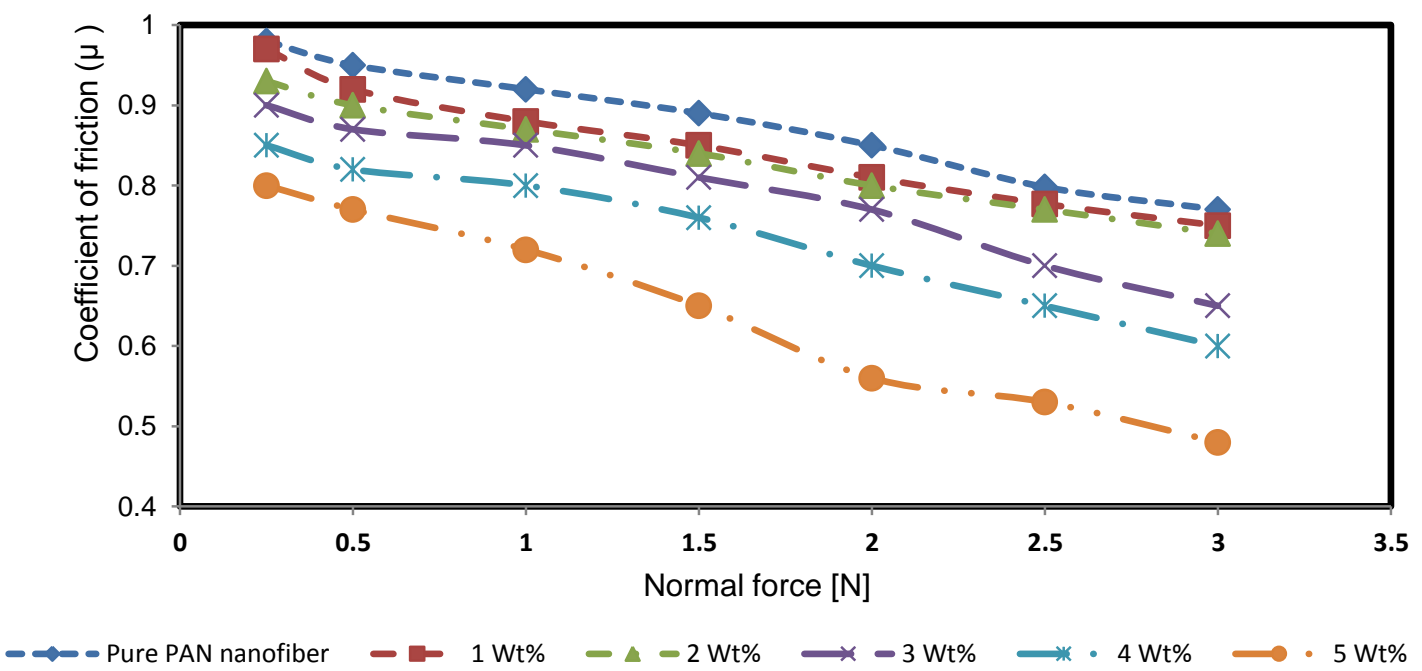

(a) 


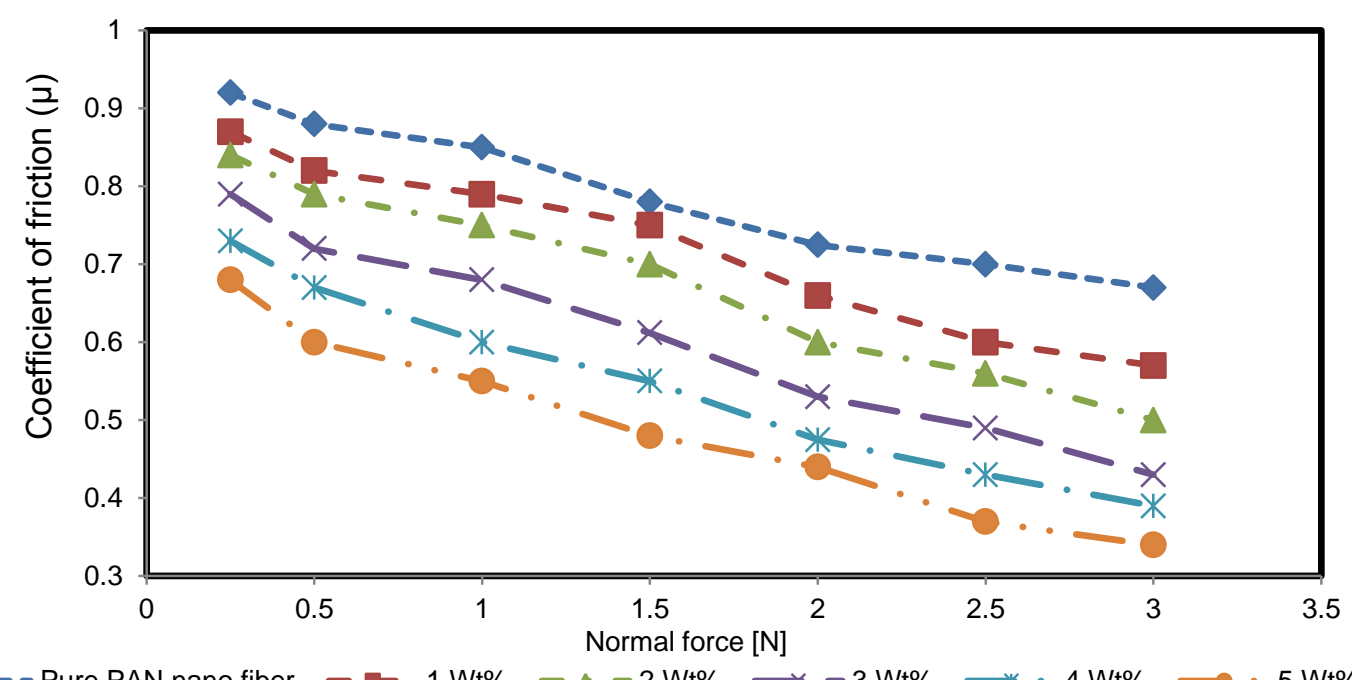

(b)

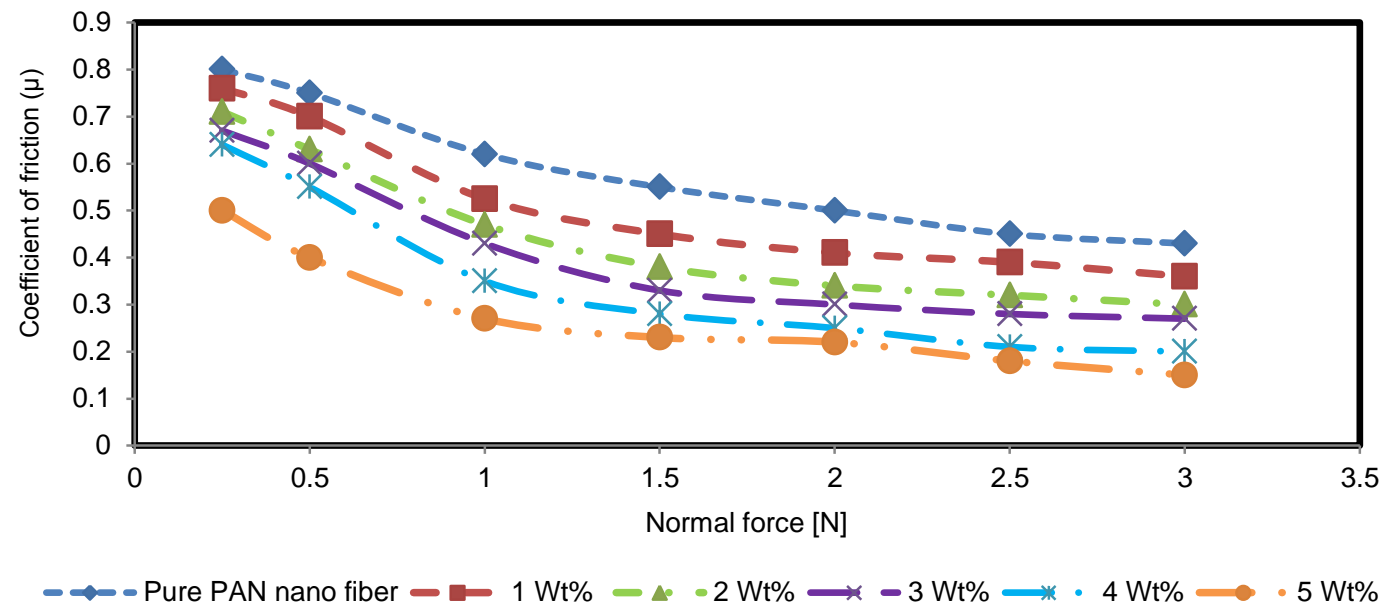

(c)

Fig. 8. Coefficient of friction of MWCNTs/PAN fabric (a) As electrospun, (b)stabilized, and (c) carbonized as a function of the normal force varying temperature of heat treatment at different wt\% of MWCNTs.

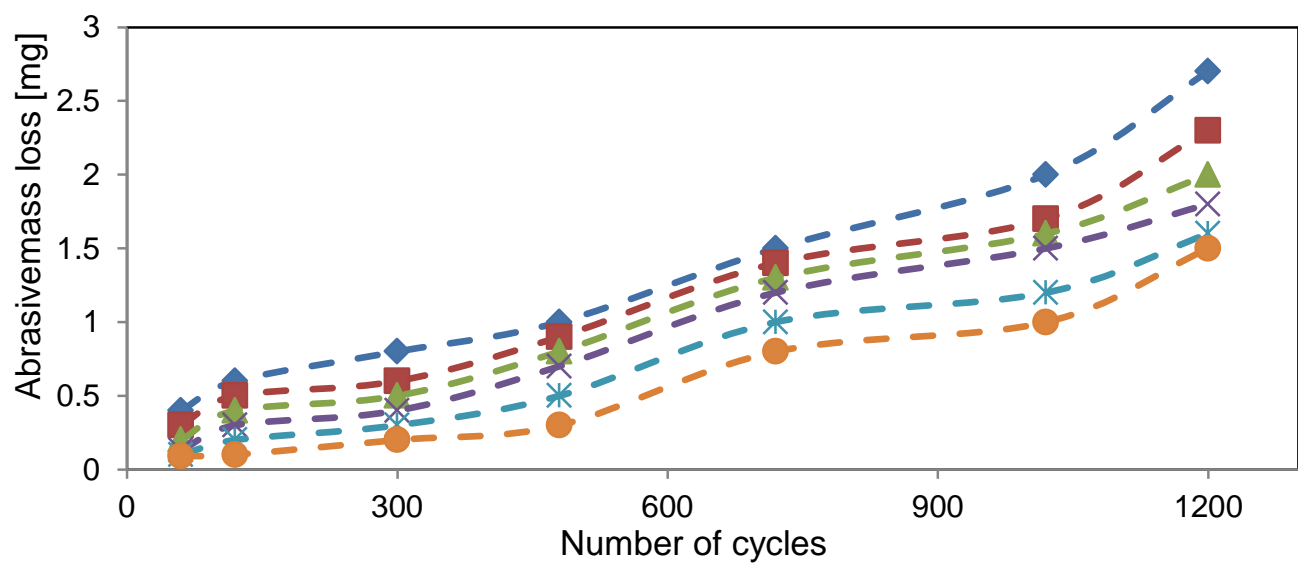

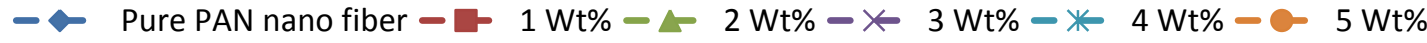

(a) 


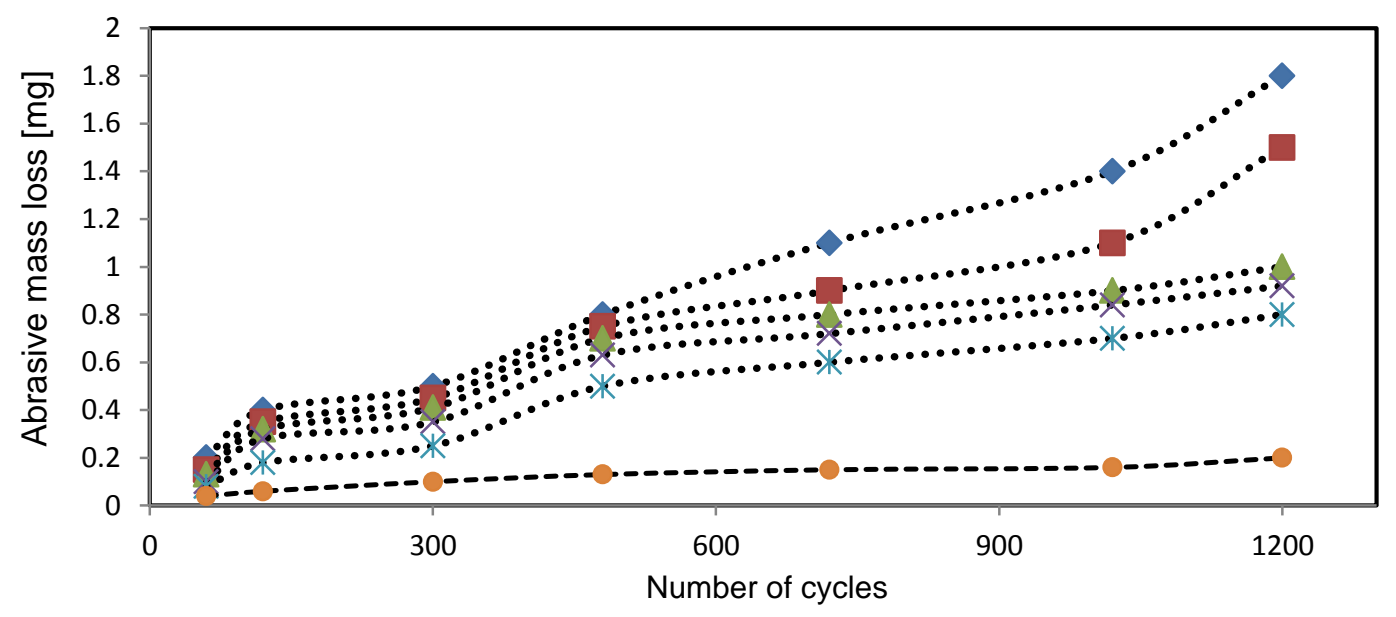

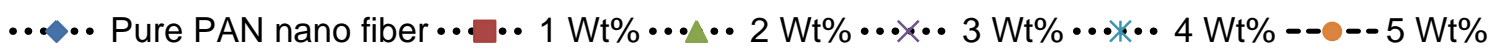

(b)

Fig. 9. Abrasive mass losses of electrospun nanofiber fabrics (a) As electrospun, and (b) carbonized as a function of number of wear cycles under $200 \mathrm{~g}$ load at Wt $\%$ vs. of MWCNTs.

\section{CONCLUSION}

We have prepared MWCNT/PAN nanofiber fabrics by using electrospinning method. The fabric was further stabilized and hot-pressed in vacuum at different temperatures. Morphological characteristics of the hot-pressed fabrics showed a decrease in average fiber diameter from $194 \mathrm{~nm} \pm 34 \mathrm{~nm}$ to $140 \mathrm{~nm} \pm 50 \mathrm{~nm}$. Also, adding MWCNTs from $1 \mathrm{wt} \%$ to $5 \mathrm{wt} \%$ decreases the average of nanofibril composite diameters and its standard deviation from $140 \mathrm{~nm} \pm 50 \mathrm{~nm}$ to $130 \mathrm{~nm} \pm 32 \mathrm{~nm}$ for the hot pressed one. HRTEM showed a formation of 2-D graphite structure. The crystallite size of such structure has been calculated by using Raman spectroscopy and showed a value of $9.6 \mathrm{~nm}$ for the hot-pressed 5wt\% MWCNTs/carbon nanofibril composite fabric which is double the reported value of commercial high modulus carbon fibers. Mechanical properties showed increases in tensile strength values from $45 \mathrm{MPa}$ to $63 \mathrm{MPa}$ for hot-pressed fabrics without and with $5 \mathrm{wt} \%$ MWCNTs respectively. For hot-pressed MWCNTs/carbon nano fibril composite fabrics a coefficient of friction as low as 0.15 and abrasive mass loss of $0.2 \mathrm{mg}$ have been reported which can be used as a good candidate for journal bearing applications. Also, coefficient of friction as high as 0.7 and abrasive mass loss of $0.75 \mathrm{mg}$ has been reported which can be used as a good candidate for braking pads.

\section{ACKNOWLEDGMENT}

Authors would like to thank prof. T.A. Eltaweel head of Production engineering and design Department, Faculty of Engineering, Menoufiya University, Egypt. Who allow for tribological characteristics in his tribology Lab. 


\section{REFERENCES}

[1] J. Doshi and D. H. Reneker, "Electrospinning process and applications of electrospun fibers," Journal of Electrostatics, vol. 35, pp. 151-160, Aug (1995).

[2] A..A. Ali and G.C. Rutledgeb, "Hot-pressed electrospun PAN nano fibers: An idea for flexible carbon mat" Journal of Materials Processing Technology, 209, pp. 4617-4620 (2009).

[3] A. A. Ali, et al." Electrical Conductivity and Dielectric Constant of Hot Pressed MWCNTs/ Carbon Nano Fibril Composite fabrics" workshop on application of advanced materials in industry, ,GUC, Egypt8th -9th June (2013).

[4] W.S. Khan, et al. "Thermal conductivities of electrospun PAN and PVP nanocomposite fibers incorporated with MWCNTs and NiZn ferrite nanoparticles" International Journal of Thermal Sciences, 71, pp. 74-79 (2013).

[5] Bo-Hye Kima,et al. "Electrochemical behavior of activated carbon nanofibervanadiumpentoxide composites for double-layer capacitors "Electrochimica Acta, 109, pp. 859- 865 (2013).

[6] Yeon-Joo Kim, et al, "Technological potential and issues of polyacrylonitrile based nanofiber non-woven separator for Li-ion rechargeable batteries" Journal of Power Sources, 244, pp. 196-206 (2013).

[7] Zhang G, Zhang C, Nardin P, Li WY, Liao H, Coddet C. "Effects of sliding velocity and applied load on the tribological mechanism of amorphous polyether- ether-ketone (PEEK)". Tribol Int; 41, pp. 79-86 (2008).

[8] Li L, Bellan LM, Craighead HG, Frey MW. "Formation and properties of nylon-6 and nylon-6/montmorillonite composite nanofibers" Polymer, 47(17),:6208-17 (2006).

[9] Pai CL, Boyce MC, Rutledge GC. "Mechanical Properties of Individual Electrospun PA 6(3)T Fibers and Their Variation with Fiber Diameter", Polymer; 52 (26): 6126-33 (2011).

[10] Silberstein MN, Pai CL, Rutledge GC, Boyce MC, "Elastic-Plastic Behavior of NonWoven Mats", Journal of the Mechanics and Physics of Solids; 60(2), pp. 295-318 (2012).

[11] Wang X, et al. "Enhanced Mechanical Performance of Self-Bundled Electrospun Fiber Yarns via Post-Treatments", Macromolecular Rapid Communications; 29(10): pp. 826-31 (2008).

[12] Lai C, Zhong G, et al." Investigation of post-spinning stretching process on morphological, structural, and mechanical properties of electrospun polyacrylonitrile copolymer nanofibers", Polymer 2011; 52(2): 519-28.

[13] $\mathrm{Na} \mathrm{H}$, et al."Effect of Hot-Press on Electrospun Poly(Vinylidene Fluo- ride) Membranes," Polymer Engineering and Science; 48(5):934-40 (2008).

[14] Jeong $L$, et al. "Time-resolved structural investigation of regenerated silk fibroin nanofibers treated with solvent vapor" International Journal of Biological Macromolecules;38(2):140-4 (2006).

[15] Cho D, Zhmayev E, Joo YL," Structural Studies of Electrospun Nylon 6 Fibers from Solution and Melt", Polymer 2010; 52:4600-9.

[16] Pai CL, Boyce MC, Rutledge GC "On the Morphology of Porous and Wrinkled Fibres of Polystyrene Electrospun from Dimethylformamide", Macromolecules; 42(6):2102-14 (2009).

[17] Derler S, Schrade GU, Gerhardt LC, "Tribology of human skin and mechanical skin equivalents in contact with textiles" Wear 2007;263:1112-6.

[18] Gerhardt LC, Mattle N, Schrade GU, Spencer ND, Derler S. Skin Research and Technology 2007; 14(1):77-88. 
[19] Guo-ming Lin, et al." Hybrid effect of nanoparticles with carbon fibers on the mechanical and wear properties of polymer composites" Composites: Part B 43 (2012) 44-49.

[20] Matthew M, C. Rutledge et al. "Mechanical and tribological properties of electrospun PA 6(3) T fiber mats" Polymer 53 (2012) 3017-3025.

[21] T. Policandriotes, P. Filip, "Effects of selected nanoadditives on the friction and wear performance of carbon-carbon aircraft brake composites". Wear 271 (2011) 2280- 2289.

[22] Chen IH, Wang CC, Chen CY,"Fabrication and structural characterization of polyacrylonitrile and carbon nanofibers containing plasma-modified carbon nanotubes by Electrospinning". J Phys Chem C 2010; 114(32):13532-9. 